\title{
Clinical utility gene card for: BEST1-related dystrophies (Bestrophinopathies)
}

\author{
Simon C Ramsden ${ }^{1}$, Alice E Davidson ${ }^{2}$, Bart P Leroy ${ }^{3,4}$, Anthony T Moore ${ }^{2,5}$, Andrew R Webster ${ }^{2,5}$, \\ Graeme CM Black ${ }^{\star 1}$ and Forbes DC Manson ${ }^{6}$
}

European Journal of Human Genetics (2012) 20, doi:10.1038/ejhg.2011.251; published online 11 January 2012

\section{DISEASE CHARACTERISTICS}

\subsection{Name of the disease (synonyms)}

Bestrophinopathy (Best disease, Best vitelliform macular dystrophy, autosomal dominant vitreoretinochoroidopathy, autosomal recessive bestrophinopathy, adult-onset vitelliform dystrophy, retinitis pigmentosa).

\subsection{OMIM\# of the disease}

Mutations in BEST1 cause a range of clinically heterogeneous retinal dystrophies (collectively termed bestrophinopathies) including; Best disease (OMIM\#153700), autosomal dominant vitreoretinochoroidopathy (ADVIRC; OMIM\#193220), autosomal recessive bestrophinopathy (ARB: OMIM\#611809), adult-onset vitelliform macular dystrophy: OMIM\#608161), and retinitis pigmentosa (RP: OMIM\#268000 and OMIM\#613194).

1.3 Name of the analysed genes or DNA/chromosome segments BEST1 (formally VMD2).

\subsection{OMIM\# of the gene(s)}

607854 .

\subsection{Mutational spectrum}

The mutational spectrum associated with the different bestrophinopathies varies greatly. To date $>100$ different BEST1 mutations, the vast majority of which are heterozygous missense variants, have been associated with Best disease. ${ }^{1}$ Currently only four distinct heterozygous missense mutations have been associated with ADVIRC. ${ }^{2,3}$ A combination of compound heterozygous or homozygous missense and nonsense mutations have now been described in $>20$ patients with $\mathrm{ARB}^{4}{ }^{4}$ Missense mutations are also associated with autosomal dominant and concentric RP, and the p.L140V homozygous missense mutation that has been associated with ARB was also causative in an apparent case of autosomal recessive RP. ${ }^{5}$ Many of the BEST1 pathogenic variants that are associated with Best disease show variable expressivity and incomplete penetrance at the clinical level. Some patients with a mutation in BEST1, which leads to Best disease, even have a normal electro-oculogram (EOG) light-rise (see section 3.1.2). ${ }^{6}$
A summary of all allelic variants reported in BEST1 can be found on a web-based database of the University of Regensburg: http://wwwhuge.uni-regensburg.de/BEST1_database/home.php?select_db=BEST1.

\subsection{Analytical methods}

Bi-directional fluorescent Sanger sequencing of coding and intron-exon boundaries of BEST1.

\subsection{Analytical validation}

There are currently no reference materials to act as positive controls, consequently, parallel bi-directional fluorescent Sanger sequencing of known controls is required to validate procedures. Diagnostic testing must be carried out within a laboratory environment working to standards compliant with the ISO 15189.

1.8 Estimated frequency of the disease (incidence at birth ('birth prevalence') or population prevalence)

The exact prevalence of Best disease and other bestrophinopathies is unknown. The majority (61\%) of BEST1 mutations have only been identified in single families. ${ }^{1}$ However, population variation has also been observed, for example, the Best disease associated mutation p.Thr6Pro has a particularly high frequency in the Netherlands, which is predicted to be the consequence of a founder effect. ${ }^{1,7,8}$

1.9 If applicable, prevalence in the ethnic group of investigated person

Unknown.

\subsection{Diagnostic setting}

\begin{tabular}{lll}
\hline & Yes & No \\
A. (Differential) diagnostics & $\Downarrow$ & $\square$ \\
B. Predictive testing & $\Downarrow$ & $\square$ \\
C. Risk assessment in relatives & $\Downarrow$ & $\square$ \\
D. Prenatal & $\Downarrow$ & $\square$
\end{tabular}

Comment: Not applicable. 


\section{TEST CHARACTERISTICS}

\begin{tabular}{|c|c|c|c|c|}
\hline & \multicolumn{2}{|c|}{ Genotype or disease } & \multirow{2}{*}{$\begin{array}{l}\text { A: True positives } \\
\text { B: False positives }\end{array}$} & \multirow{2}{*}{$\begin{array}{l}\text { C: False negatives } \\
\text { D: True negatives }\end{array}$} \\
\hline & Present & Absent & & \\
\hline \multicolumn{5}{|l|}{ Test } \\
\hline \multirow[t]{2}{*}{ Positive } & A & B & Sensitivity: & $A /(A+C)$ \\
\hline & & & Specificity: & $\mathrm{D} /(\mathrm{D}+\mathrm{B})$ \\
\hline \multirow[t]{2}{*}{ Negative } & C & D & Positive predictive value: & $A /(A+B)$ \\
\hline & & & Negative predictive value: & $D /(C+D)$ \\
\hline
\end{tabular}

\subsection{Analytical sensitivity}

(proportion of positive tests if the genotype is present)

On the basis of resequencing of positive controls, we estimate that the analytical sensitivity and specificity of the test used (bi-directional Sanger sequencing) will be $>98 \%$.

\subsection{Analytical specificity}

(proportion of negative tests if the genotype is not present)

See above. We estimate analytical specificity of $>98 \%$ given current testing methodologies.

\subsection{Clinical sensitivity}

\section{(proportion of positive tests if the disease is present)}

On the basis of published evidence and service experience, we would estimate that this test has a high clinical sensitivity, in particular in those with a high clinical index of suspicion, especially in patients with an abnormal EOG light-rise (see section 3.1.2). In an unvalidated series referred for testing within a service environment, a pathogenic variant was found in $85 \%$ of patients with a clinical suspicion of either Best disease or ARB (unpublished data).

\subsection{Clinical specificity}

\section{(proportion of negative tests if the disease is not present)}

The clinical specificity is dependent on variable factors such as age or family history. In such cases a general statement should be given, even if quantification can only be made case by case.

\subsection{Positive clinical predictive value}

\section{(life-time risk to develop the disease if the test is positive)}

Given the high penetrance of BEST1 mutations, if pathogenicity can be proven the clinical validity of the test is approaching $100 \%$; however, variable penetrance must be taken into account. Missense changes account for $\sim 60 \%$ of BEST1 mutations. The clinical relevance of all variants is assessed according to current agreed practice. However, a number of variants remain currently unclassified. Family studies may be required to aid interpretation (for example, segregation of disease in families with dominant inheritance). At least some of these unclassified variants may be counted as potential false positive results.

\subsection{Negative clinical predictive value} (probability not to develop the disease if the test is negative) For known pathogenic changes, the negative predictive value will be approaching $100 \%$. If no pathogenic change has been identified one can assume an increased risk based on family history for a non-affected person. Allelic and locus heterogeneity may need to be considered.
Index case in that family had been tested: Approaching 100\%.

Index case in that family had not been tested:

Owing to the incomplete detection rate of mutations for all BEST1related phenotypes, a negative test result in this context has questionable value.

\section{CLINICAL UTILITY}

3.1 (Differential) diagnosis: The tested person is clinically affected (To be answered if in 1.10 'A' was marked)

\subsubsection{Can a diagnosis be made other than through a genetic test?}

\begin{tabular}{|c|c|c|}
\hline No & $\square$ (continue with 3.1 .4 ) & \\
\hline \multirow[t]{7}{*}{ Yes } & $凶$ & \\
\hline & Clinically & $\otimes$ \\
\hline & Imaging & 凶 \\
\hline & Endoscopy & $\square$ \\
\hline & Biochemistry & $\square$ \\
\hline & Electrophysiology & 凶 \\
\hline & Other (please describe) & \\
\hline
\end{tabular}

\subsubsection{Describe the burden of alternative diagnostic methods to the patient?.}

For the patients with suspected Best disease a clinical diagnosis is made based on fundus examination, autofluorescence imaging, optical coherence tomography (OCT) and electrophysiology testing. Hence, pre-symptomatic testing may be undertaken by such examination.

The combination of a normal electroretinogram (ERG) and a reduced or absent EOG light-rise is specific to dominant Best disease. The EOG records the change in the electrical potential between the cornea and the retinal-pigmented epithelium using skin electrodes placed on either side of the eye. After a decrease of this standing potential during dark adaptation, there normally is an increase of it in response to light adaptation (the light-rise). The minimum amplitude of the standing potential in the dark, and the maximum in the light, is expressed as the Light Peak $/$ Dark $_{\text {trough- }}{ }^{-}$or Arden ratio. ${ }^{9}$ Arden ratios $<1.5$ are generally reported as abnormally low, and those $>2.0$ are generally reported as normal; ratios between 1.5 and 2.0 may be reported as borderline. ${ }^{9}$ An abnormal EOG light-rise is a frequent, albeit not universal finding, so that genetic testing is essential in families showing reduced penetrance. In addition, high-resolution OCT is used to identify abnormal accumulation of lipofuscin between the photoreceptors and the retinal pigment epithelium due to impaired photoreceptor outer segment metabolism. Increased lipofuscin accumulation also leads to hyperautofluorescence of the egg yolk-like fundus lesions on autofluorescence imaging using 488-nm blue light.

Best disease can be clinically similar to some cases of ARB, a recently recognised condition that cannot be diagnosed unequivocally in all cases. ARB is characterised by a severely reduced EOG light-rise in the presence of a reduced (but not absent) ERG. High-resolution OCT and autofluorescence imaging may be useful in differentiating between Best disease and ARB, and might suggest a diagnosis of ARB before genetic testing. However, in many cases a definitive clinical diagnosis can only be secured by genetic testing.

ADVIRC is an infrequent condition, with a pathognomonic hyperpigmented band in the retinal periphery. Nevertheless, when at a young age, this band is not as clearly visible on fundoscopy, an EOG 
and ERG are also required to make the diagnosis. The EOG light-rise is severely impaired before the ERG declines.

An important consideration is that electrophysiological testing cannot be performed easily in young children (although an ERG is possible, an EOG needs a co-operative child). Consequently, molecular diagnostic testing is of particular importance to confirm the diagnosis in children.

\subsubsection{How is the cost effectiveness of alternative diagnostic methods} to be judged?.

Bestrophinopathies are associated with severe and often early-onset visual disability and blindness, secondary to retinal degeneration. The group of conditions is therefore associated with a significant and progressive disability in the context of normal general health that is also progressive.

Genetic testing for BEST1 mutations provides a precise molecular diagnosis. This provides information regarding recurrence risk, carrier status and hence provides choices that would not otherwise be available.

The inherited macular dystrophies are a group of conditions that are difficult to distinguish clinically. Gene testing is essential in defining inheritance patterns and enabling effective genetic counselling. A positive genetic test will preclude the need for further genetic testing (for example, RDS/peripherin). Lack of genetic testing may result in inaccurate diagnosis and leave an uncertainty with regard to providing detailed information on risk to future children and to other family members. The conditions include those of recessive and dominant inheritance, which are often difficult to distinguish clinically. Identification of one or two BEST1 mutation(s) will prevent further genetic and clinical investigations.

3.1.4 Will disease management be influenced by the result of a genetic test?

\begin{tabular}{lll}
\hline No & $\square$ \\
Yes & $\begin{array}{l}\text { Therapy (please } \\
\text { describe) } \\
\text { Prognosis (please } \\
\text { describe) } \\
\text { Management } \\
\text { (please describe) }\end{array}$ & No \\
& $\begin{array}{l}\text { Additionally angle-closure glaucoma is commonly } \\
\text { associated with the bestrophinopathies and often } \\
\text { contributes to visual loss incurred by patients. We } \\
\text { therefore recommend that all bestrophinopathy } \\
\text { patients should be screened for angle-closure glau- } \\
\text { coma once diagnosed. }\end{array}$ \\
\hline
\end{tabular}

3.2 Predictive setting: The tested person is clinically unaffected but carries an increased risk based on family history

(To be answered if in 1.10 ' $\mathrm{B}$ ' was marked)

\subsubsection{Will the result of a genetic test influence lifestyle and prevention?.}

If the test result is positive (please describe):

The result may influence choice of career and inform for family planning.

If the test result is negative (please describe):

The result may influence choice of career and inform for family planning.
3.2.2 Which options in view of lifestyle and prevention does a person at-risk have if no genetic test has been done (please describe)?.

As most of the patients with a bestrophinopathy suffer from considerable loss-of-vision either during their teenage years or at the latest during their active adult life, professions requiring perfect vision are impossible. Hence, a clinically confirmed diagnosis can already help in providing guidance regarding career choice.

3.3 Genetic risk assessment in family members of a diseased person (To be answered if in 1.10 'C' was marked)

\subsubsection{Does the result of a genetic test resolve the genetic situation in} that family?.

As the phenotype of ARB and Best disease can be quite similar, a molecular diagnosis in an affected individual in the context of no affected parents can confirm whether the inheritance pattern is either autosomal recessive or autosomal dominant with reduced penetrance.

3.3.2 Can a genetic test in the index patient save genetic or other tests in family members?.

Where molecular testing has identified a cause, it will be possible to screen family members at the molecular level rather than using EOG testing, which is cumbersome for the patient and less reliable.

3.3.3 Does a positive genetic test result in the index patient enable a predictive test in a family member?

Yes.

3.4 Prenatal diagnosis

(To be answered if in 1.10 'D' was marked)

3.4.1 Does a positive genetic test result in the index patient enable a prenatal diagnostic?.

Yes.

\section{IF APPLICABLE, FURTHER CONSEQUENCES OF TESTING}

Please assume that the result of a genetic test has no immediate medical consequences. Is there any evidence that a genetic test is nevertheless useful for the patient or his/her relatives? (Please describe)

Genetic testing for BEST1 mutations will provide a precise molecular diagnosis. This yields information regarding recurrence risk, carrier status and hence will provide choices that would not otherwise be available to facilitate decision making for the patient and their family. The inherited macular dystrophies are a group of conditions that are difficult to distinguish clinically. Gene testing is essential in defining inheritance patterns and enabling effective genetic counselling. A positive gene test will preclude the need for further genetic testing (for example, RDS/peripherin).

\section{CONFLICT OF INTEREST}

The authors declare no conflict of interest.

\section{ACKNOWLEDGEMENTS}

This work was supported by the EuroGentest2 (Unit 2: 'Genetic testing as part of health care'), a Coordination Action under FP7 (Grant Agreement Number 261469) and the European Society of Human Genetics. AED, SCR and GCMB gratefully acknowledge the support of RP Fighting Blindness.BPL is a Senior Clinical Investigator of the Research Foundation - Flanders (FWO; Belgium), and is further supported by FWO Flanders Grant OZP $3 \mathrm{G} 004306$. 
1 Boon CJ, Jeroen Klevering B, Leroy BP, Hoyng CB, Keunen JE, den Hollander AI: The spectrum of ocular phenotypes caused by mutations in the BEST1 gene. Prog Retin Eye Res 2009; 28: 187-205.

2 Yardley J, Leroy BP, Hart-Holden $\mathrm{N}$ et al: Mutations of VMD2 splicing regulators cause nanophthalmos and autosomal dominant vitreoretinochoroidopathy (ADVIRC). Invest Ophthalmol Vis Sci 2004; 45: 3683-3689.

3 Burgess R, Maclaren R, Davidson A et al: ADVIRC is caused by distinct mutations in BEST1 that alter pre-mRNA splicing. J Med Genet 2008; 46: 620-625.

4 Burgess R, Millar ID, Leroy BP et al: Biallelic mutation of BEST1 causes a distinct retinopathy in humans. Am J Hum Genet 2008; 82: 19-31.

5 Davidson AE, Millar ID, Urquhart JE et al: Missense mutations in a retinal pigment epithelium protein, bestrophin-1, cause retinitis pigmentosa. Am J Hum Genet 2009, 85: 581-592.
6 Testa F, Rossi S, Passerini I et al: A normal electro-oculography in a family affected by best disease with a novel spontaneous mutation of the BEST1 gene. Br J Ophthalmol 2008; 92: 1467-1470.

7 Boon CJ, Klevering BJ, den Hollander Al et al: Clinical and genetic heterogeneity in multifocal vitelliform dystrophy. Arch Ophthalmol 2007; 125: 1100-1106.

8 Petrukhin K, Koisti MJ, Bakall B et al: Identification of the gene responsible for Best macular dystrophy. Nat Genet 1998; 19: 241-247.

9 Marmor MF, Brigell MG, McCulloch DL, Westall CA, Bach M: International Society for Clinical Electrophysiology of Vision (2011). 'ISCEV standard for clinical electrooculography (2010 update). Doc Ophthalmol 2011; 122: 1-7.

10 Davidson AE, Sergouniotis PI, Burgess-Mullan R et al: A synonymous codon variant in two patients with autosomal recessive bestrophinopathy alters in vitro splicing of BEST1. Mol Vis 2010; 16: 2916-2922. 